\title{
Title: Crystallization \& Order of Small Molecules and Nucleic Acids \& their Protein Complexes
}

Susanna Huang * and Lillian Hu, Department of Biomolecular Crystallography,

SeNA Research Institute., Atlanta, Georgia; email: Se_Nucleic_Acids@senaresearch.org

In order to prevent the growth of cancer cells and viruses, their molecular information and signal carriers (such as nucleic acids and proteins) need be stopped. When specific structures of disease-causing DNA \& RNA and their protein complexes are determined, drugs can be developed to selectively target viral \& cancerous nucleic acids and their protein complexes to prevent the processes of the replication, transcription and/or translation, eventually treating diseases effectively. In order to determine the structures of nucleic acids through X-ray diffraction and/or neutron diffraction, scientists must grow large crystals of DNA, RNA and/or their protein complexes. However, there are many challenges in macromolecular crystallization (especially nucleic acids and protein complexes) that can prevent efficient and rapid packing of these bio-macromolecules. These challenges include: (1) repelling, negatively charged phosphates that cause disruption in regular molecular packing; (2) sample purity and molecular chemical-compositional heterogeneity; (3) different multiple conformations, namely molecular conformational heterogeneity; (4) irregular nucleic acid structures and shapes, making molecular order challenging; (5) molecular and crystal dynamics that make the formation of well-defined structures and order difficult, making molecular packing extremely challenging.

Recently, we have discovered that the incorporation of selenium into nucleic acids may largely solve these above problems, resulting in larger high-quality crystals and higher diffraction resolution. Therefore, we have hypothesized that the selenium incorporation and derivatization can facilitate the molecular packing and high-resolution diffraction of DNA, RNA and their protein complexes in many different ways. These may include (1) larger delocalization of the phosphate charges, thus reducing the negative charge repulsion; (2) increase of the chemical synthesis efficiency \& purity and polymerization fidelity \& cleanliness, thus decreasing molecular chemical heterogeneity; (3) increase of the intra-molecular interactions of nucleic acid strands, thus enhancing molecular conformational homogeneity; (4) facilitation of inter-molecular interactions, thus strengthening molecular packing of irregularly-shaped nucleic acids and their protein complexes; (5) facilitation of intra- and inter-molecular interactions, thus reducing molecular \& crystal dynamics and enhancing crystal packing.

Through observations of crystal growth at different time intervals, we discovered that with selenium derivatization, the packing and crystallization of the biomolecules with Se are much more rapid than with corresponding natives. In addition, the Se crystals are normally much larger than the native crystals. For an example, the crystals with selenium were $\mathbf{6 0 0}$ microns in size, while the corresponding natives without selenium were approximately 60 microns in size. In conclusion, our hypothesis is supported by our experiments and observations: The incorporation of selenium atoms may largely facilitate the high-quality crystallization and high-resolution diffraction of nucleic acids and their protein complexes. We will present our exciting discoveries in this conference. Work is supported by NIH ES026935.

\section{Selected Publications:}

Venu Gopal Vandavasi, Matthew P. Blakeley, David A. Keen, Lillian R. Hu, Zhen Huang and Andrey Kovalevsky, "Temperature-induced replacement of phosphate proton with metal ion captured in neutron structures of A-DNA”, Structure, 2018, 26, 1-6. 